\title{
The Pan-European Mobile Radio System Part II
}

\author{
L. Hanzo, R. Steele \\ Department of Electronics and Computer Science, University of Southampton \\ Highfield S09 5NH Southampton - UK
}

\begin{abstract}
Having considered the mapping of logical traffic and control channels onto physical channels, speech and error correction coding, interleaving as well as the TDMA hierarchy and synchronisation problems in Part I of this contribution, here mainly transmission issues are addressed [1 - 6] and some performance figures are provided. Constant envelope partial response Gaussian Minimum Shift Keying (GMSK) with a channel spacing of $200 \mathrm{kHz}$ is deployed to support 125 duplex channels in the $890-915 \mathrm{MHz}$ uplink and $935-960 \mathrm{MHz}$ downlink bands. respectively. At a transmission rate of $271 \mathrm{kbit} / \mathrm{s} 1.35 \mathrm{bit} / \mathrm{s} / \mathrm{Hz}$ spectral efficiency is achieved. The controlled GMSK-induced and un-controlled channel-induced inter-symbol interferences are removed by the channel equaliser. The set of standardised wide-band GSM channels is introduced in order to provide bench-markers for performance comparisons. Efficient power budgeting and minimum cochannel interferences are ensured by the combination of adaptive power- and handover-control based on weighted averaging of up to eight uplink and downlink system parameters. Discontinuous transmissions (DTX) assisted by reliable spectral-domain voice activity detection (VAD) and comfor-noise insertion further reduce interferences and power consumption. Due to ciphering, no unprotected information is sent via the radio link. As a result, spectrally efficient, high-quality mobile communications with a variety of services and international roaming is possible in cells of up to $35 \mathrm{~km}$ radius for signal to noise- and interference-ratios in excess of $10-12 \mathrm{dBs}$.
\end{abstract}

\section{INTRODUCTION}

As an organic extension of Part I, where the construction of speech, data and signalling channels was considered, in this treatise we focus on transmission issues of the GSM system. The channel coded information of eight users is multiplexed to yield a stream of about 271 $\mathrm{kbit} / \mathrm{s}$, which is transmitted using constant envelope Gaussian minimum shift keying (GMSK) modulation [6]. The system operates under wide-band conditions in the range of $890-915 \mathrm{MHz}$ for uplink and 935-960 $\mathrm{MHz}$ for downlink directions, using 125 paired duplex channels with $200 \mathrm{kHz}$ channel spacing in each band with a spectral efficiency of $1.35 \mathrm{bit} / \mathrm{s} / \mathrm{Hz}$.

In subsequent sections we briefly consider aspects of GMSK modulation based on Recommendation [R05.04.] in section 2 and the associated problems of wideband transmissions and the standardised set of wideband GSM channel models [R05.05] in section 3. Although the equalisation algorithm itself is not specified, its required performance is. The potentially best scheme is the maximum likelihood Viterbi equalisation

(VE) discussed in section 4. Adaptive radio link control [R.05.08], discontinuous transmission [R.06.31.] (DTX), voice activity detection [R.06.32] (VAD) and comfort noise insertion (CNI) along with ciphering issues [R.03.20.] are going to be highlighted in sections $5-8$, respectively. System performance requirements based on [R.05.05.] and some experimental performance figures are presented in section 9 , while a parameter summary is given in section 10 .

\section{GLOSSARY}

A3

A5

A8

$A B$

$\mathrm{ACCH}$

$\mathrm{ADC}$

$\mathrm{AGCH}$

AUC
Authentication algorithm Cyphering algorithm Confidential algorithm to compute the cyphering key

Access burst

Associated control channel

Administration centre

Access grant control channel

Authentication centre 


\begin{tabular}{|c|c|c|c|}
\hline AWGN & Additive gaussian noise & \multirow[t]{3}{*}{ MAIO } & \multirow{2}{*}{$\begin{array}{l}\text { Mobile allocation index offset: } \\
\text { initial RF channel offset, input }\end{array}$} \\
\hline $\mathrm{BCCH}$ & Broadcast control channel & & \\
\hline BER & Bit error ratio & & variable of the FH algorithm \\
\hline BFI & Bad frame indicator flag & MS & Mobile station \\
\hline BN & Bit number & MSC & Mobile switching centre \\
\hline BS & Base station & MSRN & Mobile station roaming number \\
\hline BS-PBGT & $\begin{array}{l}\text { BS power budget to be evaluat- } \\
\text { ed for power budget motivated } \\
\text { handovers }\end{array}$ & MS-TXPWR-MAX & $\begin{array}{l}\text { Maximum permitted MS trans- } \\
\text { mitted power on a specific traf- } \\
\text { fic channel in a specific traffic }\end{array}$ \\
\hline BSIC & Base station identifier code & & cell \\
\hline $\mathrm{CC}$ & Convolutional codec & \multirow{3}{*}{ MS-TXPWR-MAX(n) } & Maximum permitted MS trans- \\
\hline $\mathrm{CCCH}$ & Common control channel & & mitted power on a specific \\
\hline \multirow[t]{2}{*}{ CELL-BAR-ACCESS } & $\begin{array}{l}\text { Boolean flag to indicate, wheth- } \\
\text { er the MS is permitted to access }\end{array}$ & & $\begin{array}{l}\text { channel in the } n \text {-th adjacent } \\
\text { traffic cell }\end{array}$ \\
\hline & the specific traffic cell & NB & Normal burst \\
\hline $\mathrm{CNC}$ & Comfort noise computation & NMC & Network management centre \\
\hline CNI & Comfort noise insertion & NUFR & Receiver noise update flag \\
\hline $\mathrm{CNU}$ & $\begin{array}{l}\text { Comfort noise update state in } \\
\text { the DTX handler }\end{array}$ & NUFT & $\begin{array}{l}\text { Noise update flag to ask for SID } \\
\text { frame transmission }\end{array}$ \\
\hline DB & Dummy burst & OMC & Operation and maintenance \\
\hline DL & Down link & & centre \\
\hline \multirow[t]{2}{*}{ DSI } & Digital speech interpolation to & PARCOR & Partial correlation \\
\hline & improve link efficiency & $\mathrm{PCH}$ & Paging channel \\
\hline \multirow[t]{2}{*}{ DTX } & Discontinuos transmission for & PCM & Pulse code modulation \\
\hline & $\begin{array}{l}\text { power consumption and inter- } \\
\text { ference reduction }\end{array}$ & PIN & $\begin{array}{l}\text { Personal identity number for } \\
\text { MSs }\end{array}$ \\
\hline EIR & Equipment identity register & PLMN & Public land mobile network \\
\hline EOS & $\begin{array}{l}\text { End of speech flag in the DTX } \\
\text { handler }\end{array}$ & PLMN-PERMITTED & $\begin{array}{l}\text { Boolean flag to indicate, wheth- } \\
\text { er the MS is permitted to access }\end{array}$ \\
\hline $\mathrm{FACCH}$ & Fast associated control channel & & the specific PLMN \\
\hline FCB & Frequency correction burst & \multirow[t]{2}{*}{ PSTN } & \multirow{2}{*}{$\begin{array}{l}\text { Public switched telephone net- } \\
\text { work }\end{array}$} \\
\hline $\mathrm{FCCH}$ & Frequency correction channel & & \\
\hline FEC & Forward error correction & QN & Quarter bit number \\
\hline $\mathrm{FH}$ & Frequency hopping & \multirow[t]{2}{*}{$R$} & \multirow{2}{*}{$\begin{array}{l}\text { Random number in the authen- } \\
\text { tication process }\end{array}$} \\
\hline FN & TDMA frame number & & \\
\hline GMSK & Gaussian minimum shift keying & \multirow[t]{2}{*}{ RA } & \multirow{2}{*}{$\begin{array}{l}\text { Rural area channel impulse re- } \\
\text { sponse }\end{array}$} \\
\hline GP & Guard space & & \\
\hline HGO & Hangover in the VAD & $\mathrm{RACH}$ & Random access channel \\
\hline HLR & Home location register & $R F$ & Radio frequency \\
\hline $\mathrm{HO}$ & Handover & $R F C H$ & Radio frequency channel \\
\hline $\mathrm{HOCT}$ & Hangover counter in the VAD & \multirow[t]{2}{*}{ RFN } & \multirow{2}{*}{$\begin{array}{l}\text { Reduced TDMA frame number: } \\
\text { equivalent representation of the } \\
\text { TDMA frame number, which is }\end{array}$} \\
\hline HO-MARGIN & $\begin{array}{l}\text { Handover margin to facilitate } \\
\text { hysteresis }\end{array}$ & & \\
\hline \multirow[t]{2}{*}{ HSN } & $\begin{array}{l}\text { Hopping sequence number: fre- } \\
\text { quency hopping algorithm's in- }\end{array}$ & \multirow{3}{*}{ RTNTABLE } & $\begin{array}{l}\text { used in the synchronisation } \\
\text { channel }\end{array}$ \\
\hline & put variable & & \multirow{2}{*}{$\begin{array}{l}\text { Random number table utilised } \\
\text { in the frequency hopping algo- } \\
\text { rithm }\end{array}$} \\
\hline IMSI & $\begin{array}{l}\text { International mobile subscriber } \\
\text { identity }\end{array}$ & & \\
\hline ISDN & Integrated services digital network & RPE & Regular pulse excited \\
\hline LAI & Location area identifier & RPE-LTP & Regular pulse exciced codec \\
\hline LAR & Logarithmic area ratio & & with long term predictor \\
\hline LTP & Long term predictor & \multirow[t]{2}{*}{ RS-232 } & \multirow{2}{*}{$\begin{array}{l}\text { Serial data transmission stan- } \\
\text { dard equivalent to CCITT V24, } \\
\text { interface }\end{array}$} \\
\hline \multirow[t]{2}{*}{ MA } & $\begin{array}{l}\text { Mobile allocation: set of legiti- } \\
\text { mate RF channels, input vari- }\end{array}$ & & \\
\hline & $\begin{array}{l}\text { able in the frequency hopping } \\
\text { algorithm }\end{array}$ & RXLEV & $\begin{array}{l}\text { Received signal level: parame- } \\
\text { ter used in hangovers }\end{array}$ \\
\hline MAI & $\begin{array}{l}\text { Mobile allocation index: output } \\
\text { variable of the FH algorithm }\end{array}$ & RXQUAL & $\begin{array}{l}\text { Received signal quality: param- } \\
\text { eter used in hangovers }\end{array}$ \\
\hline
\end{tabular}




\section{The Pan-European Mobile Radio System Part II}

$\mathrm{SACCH}$

SB

$\mathrm{SCH}$

SCPC

$\mathrm{SDCCH}$

SE

SID

SIM

SPRX

SPTX

\section{STP}

TA

TB

$\mathrm{TCH}$

$\mathrm{TCH} / \mathrm{F}$

$\mathrm{TCH} / \mathrm{F} 2.4$

$\mathrm{TCH} / \mathrm{F} 4.8$

TCH/F9.6

TCH/FS

$\mathrm{TCH} / \mathrm{H}$

$\mathrm{TCH} / \mathrm{F} 2.4$

TCH/F4.8

TDMA

TMSI

TN

TU

TXFL

UL

VAD

VE

VLR
Signed response in the authentication process

Slow associated control channel

Synchronisation burst

Synchronisation channel

Single channel per carrier

Stand-alone dedicated control channel

Speech extrapolation

Silence identifier

Subscriber identity module in MSs

Speech received flag

Speech transmit flag in the D

TX handler

Short term predictor

Timing advance

Tailing bits

Traffic channel

Full-rate traffic channel

Full-rate $2.4 \mathrm{kbit} / \mathrm{s}$ data traffic channel

Full-rate $4.8 \mathrm{kbit} / \mathrm{s}$ data traffic channel

Full-rate $9.6 \mathrm{kbit} / \mathrm{s}$ data traffic channel

Full-rate speech traffic channel

Half-rate traffic channel

Half-rate $2.4 \mathrm{kbit} / \mathrm{s}$ data traffic channel

Half-rate $4.8 \mathrm{kbit} / \mathrm{s}$ data traffic channel

Time division multiple access

Temporary mobile subscriber identifier

Time slot number

Typical urban channel impulse response

Transmit flag in the DTX handier

Up link

Voice activity detection

Viterbi equaliser

Visiting location register

\section{MODULATION}

The GSM system uses GMSK modulation specified in Recommendation [R.05.04.]. Constant envelope, continuous phase modulation schemes are robust against signal fading as well as interference and have good spectral efficiency. The slower and smoother are the phase changes, the better is the spectral efficiency, since the signal is allowed to change less abruptly. However, the effect of an input bit is spread over several bit periods, leading to a so-called partial response system, which requires a channel equaliser in order to remove this controlled, deliberate intersymbol interference (ISI) even in the absence of uncontrolled channel dispersion.

Spreading phase changes with zero initial and final slopes over a number of modulation intervals yields a partial response system. The widely deployed Gaussian Minimum Shift Keying (GMSK) is derived from the full response Minimum Shift Keying (MSK) scheme. In MSK the phase changes between adjacent bit periods are piecewise linear, which results in discontinuous phase derivative, i.e., instantaneous frequency, and hence widens the spectrum. However, smoothing the phase by a filter having a Gaussian impulse response, which is known to have a minimum bandwidth, this problem is circumvented, as seen in Fig. 1, where the GMSK signal is generated by modulating and adding two quadrature carriers. The key parameter of GMSK in controlling both bandwidth and interference resistance is the $3 \mathrm{~dB}$-bandwidth - bit interval product $(\mathrm{B} \cdot \mathrm{T})$ referred to as normalised bandwidth. It was found that as the $\mathrm{B} \cdot \mathrm{T}$ product is increased from 0.2 to 0.5 , the interference resistance is improved by approximately $2 \mathrm{~dB}$ at the cost of increased bandwidth occupancy, and best compromise was achieved for $\mathrm{B} \cdot \mathrm{T}=0.3$. The spectral efficiency gain due to higher interference tolerance and hence more dense frequency reuse was found to be more significant than the spectral loss caused by wider GMSK spectral lobes.

The channel separation at the TDMA burst-rate of $271 \mathrm{kbit} / \mathrm{s}$ is $200 \mathrm{kHz}$ and the modulated spectrum must be $40 \mathrm{~dB}$ down at both adjacent carrier frequencies. When TDMA bursts are transmitted in an on-off keyed mode, further spectral spillage arises, which is mitigated

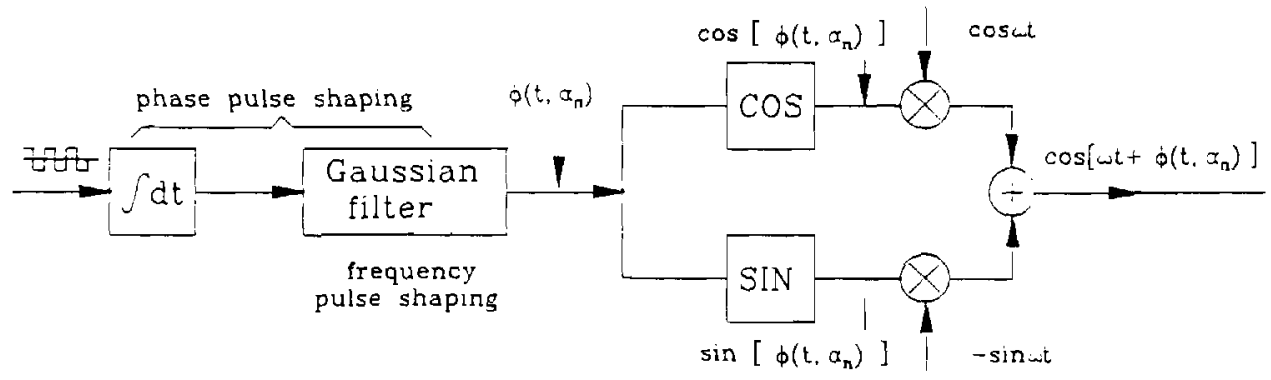

Fig. 1 - GMSK modulator schematic diagram. 


\section{Hanzo, R. Steele}

by a smooth $70 \mathrm{~dB}$ power ramp-up and down at the leading and trailing edges of the transmission bursts during a $28 \mu$ s and $18 \mu$ s interval, respectively.

\section{WIDEBAND CHANNEL MODELS}

The GSM Recommendation [R.05.05.] specifies a set of typical wideband channel impulse responses for urban, rural and hilly environments, as well as an artificially contrived equaliser test response, which will be highlighted in this section.

If the transmission bandwidth is narrower than the channel's coherence bandwidth $\left(B_{c}\right)$, all transmitted frequency components encounter nearly identical propagation delays, i.e., the so-called narrow band condition is met and the signal is subjected to non-selective or flat

TYPICAL UABAN (TU) IMPULGE RESPONSE

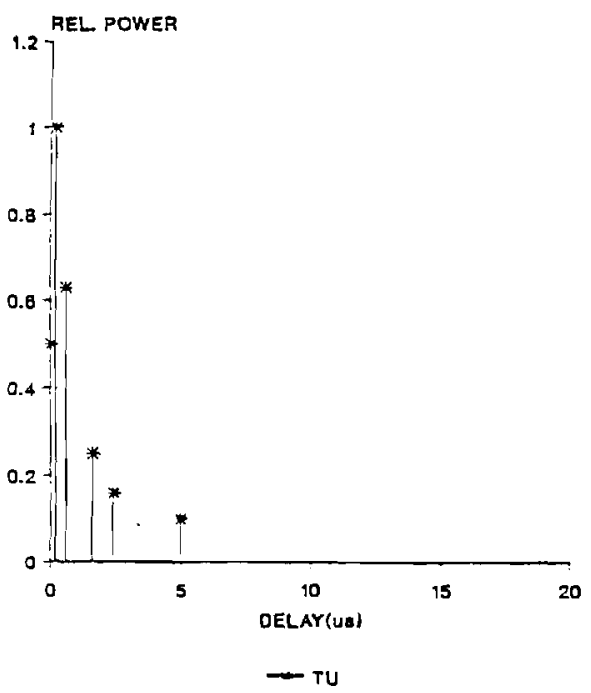

RURAL AREA (RA) IMPULSE RESPONSE

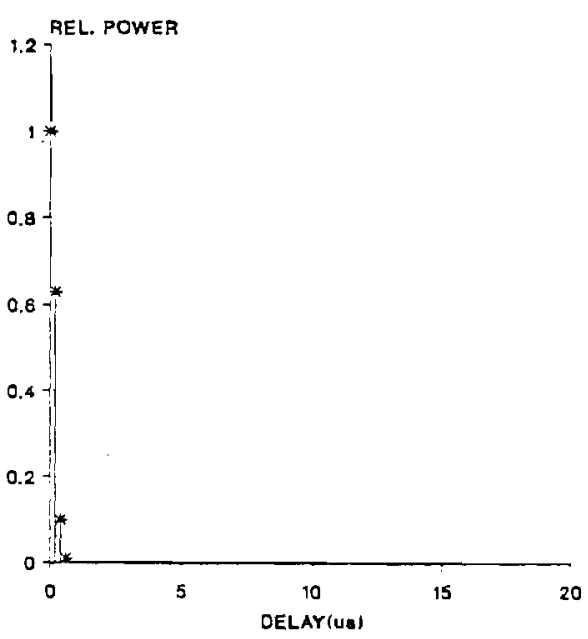

envelope fading. When the signal bandwidth is increased, for example, to accommodate several TDMA time slots as in the GSM system, the channel becomes more dispersive which results in intersymbol interference. The channel's coherence bandwidth $\left(B_{c}\right)$ is defined as the frequency, where the correlation of two received signal components' attenuation becomes less than 0.5 and $\left(B_{c}\right)$ is inversely proportional to the delay-spread $(d)$, i.e., $B_{c}=1 / 2 \pi d$.

The wideband propagation channel is the superposition of a number of dispersive fading paths, suffering from various attenuations and delays, aggravated by the phenomenon of Doppler shift caused by the MS's movement. The maximum Doppler shift $\left(f_{D} \max \right)$ is given by $f_{D} \max =v / \lambda_{c}=v \cdot f_{c} / c$, where $v$ is the vehicular speed, $\lambda_{c}$ is the wavelength of the carrier frequency $f_{c}$ and $c$ is the velocity of light. The momentary

HILLY TEARAIN (HT) IMPULSE REGPONSE

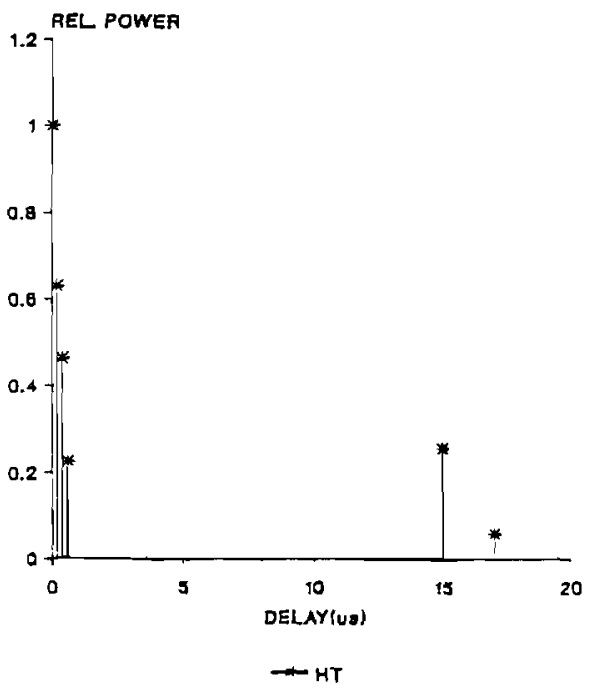

EQUALISER TEST(EQ) IMPULSE RESPONSE

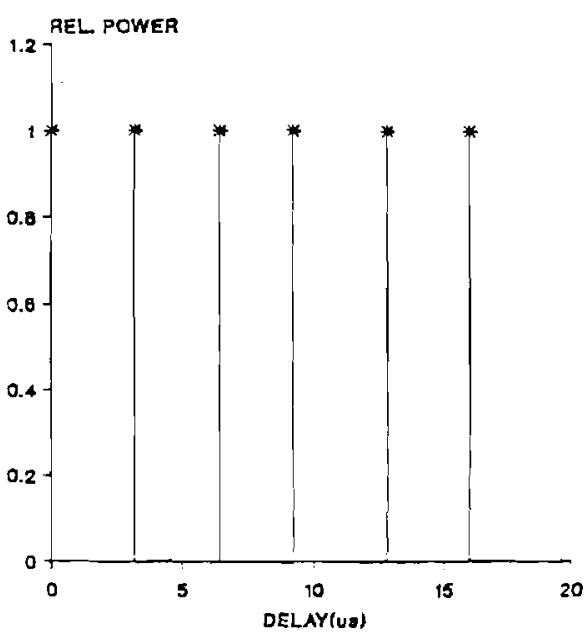

Fig. 2 - Typical GSiM channel impulse responses. 


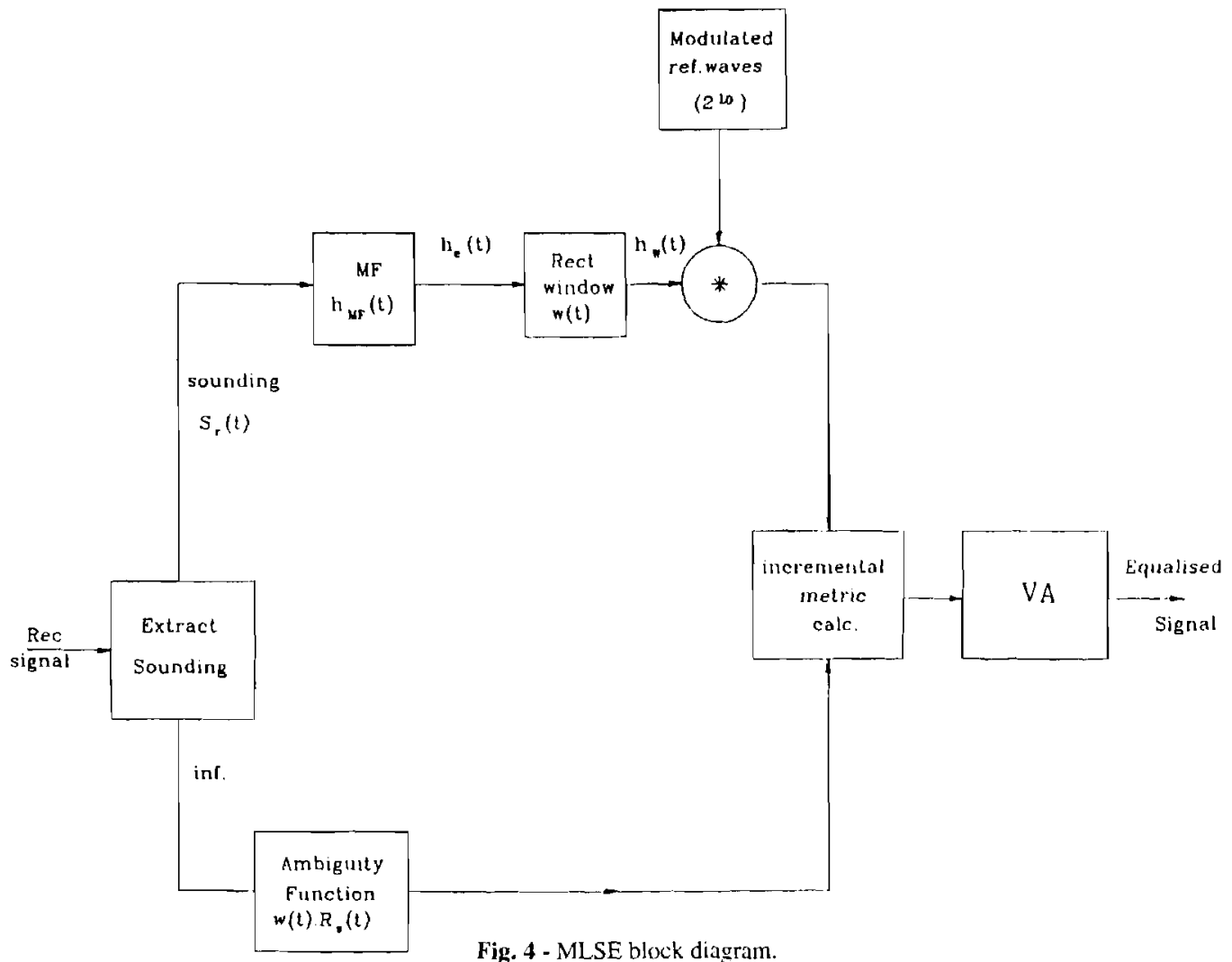

Fig. 4 - MLSE block diagram.

possible $2^{16}$ sequences. Favourable are those sequences, which have the highest autocorrelation function mainto side-lobe ratio with near-zero values around the sampling instants $\pm T, \pm 2 T$, $\pm 3 T$, $\pm 4 T$, etc., when quasiperiodically extended at both ends. It is highly desirable that both MSs and BSs use the same VE to keep development and production costs low, which additionally requires the recognition of synchronisation (SB) and access bursts (AB) as well, where 64- and 41 bit long synchronisation words are used, respectively. However, for the sake of simplicity, we only consider NBs with 26 bit midambles.

The modulated NB with the channel sounding sequence $s(t)$ in its centre is convolved with the channel's impulse response $h_{c}(t)$ and corrupted by noise.

Neglecting the noise for simplicity, the received sounding sequence becomes:

$$
s_{r}(t)=s(t) \cdot h_{c}(t)
$$

which is then matched-filtered using the impulse response $h_{M F}(t)$ to derive an estimate of the channel's impulse response:

$$
\begin{aligned}
h_{e}(t)= & s_{r}(t) \cdot h_{M F}(t)=s(t) \cdot h_{c}(t) \cdot h_{M F}(t)= \\
& R_{s}(t) \cdot h_{c}(t)
\end{aligned}
$$

where $R_{s}(t)$ is the sounding sequence's autocorrelation function. Clearly, if $R_{s}(t)$ is a highly peaked Dirac im- pulse-like function, then its convolution with $h_{c}(t)$ becomes $h_{e}(t) \approx h_{c}(t)$. With $s(t)$ in the middle of the NB the estimated $h_{c}(t)$ is quasi-stationary for the $0.577 \mathrm{~ms}$ burst duration, and can be used to equalise the 114 bits of useful information on both sides of it, although the time-variant channel precipitates higher error rates towards the burst edges. Since the complexity of the VE grows exponentially with the number of signalling intervals in the legitimate modulated reference sequences generated from all possible transmitted sequences for metric comparisons, the estimated channel response $h_{c}(t)$ has to be windowed to a computationally manageable length, while having sufficiently long memory to compensate for the typical impulse responses of the previous section.

Specifically, in addition to the duration $L_{\text {CISI }}$ of the controlled ISI, also the channel's delay-spread $L_{c}$ has to be considered in calculating the required observation interval $L_{o}=L_{\text {CISI }}+L_{c}$ of the $2^{L_{o-1}}$-state VE. In practical terms, using a bit interval of $3.69 \mu$ s and maximum channel impulse durations of around 15-20 $\mu \mathrm{s}$, a VE with a memory of 4-6 bit intervals is a good compromise, where $h_{c}(t)$ is retained over that 4-6 bit interval of its total support length, where it is exhibiting the highest energy. $L_{o}$ consecutive transmitted bits give rise to $2^{L_{o}}$ possible transmitted sequences, which are first input to a local modulator to generate the modulated waveforms, and then convolved with the windowed estimated channel response $h_{w}(t)$ to derive the legitimate reference waveforms for metric calculation, as portrayed in Fig. 4. 
Recall that $h_{e}(t)=h_{c}(t)$ only, if $R_{s}(t)$ is the Dirac delta function, which is not possible when finite-length sounding sequences are used. The true channel response $h_{c}(t)$ could only be computed by deconvolution from eq. 2 upon neglecting the rectangular window $w(t)$. Alternatively, the received signal can be convolved for the sake of metric calculation with the windowed autocorrelation function $w(t) \cdot R_{s}(t)$ often referred to as ambiguity function, which is more practical. This filtered signal is then compared to all possible reference signals and the incremental metrics $m_{i}, i=0 \ldots\left(2^{L_{0}-1}\right)$ are computed, which are utilised by the Viterbi algorithm (VA) to determine the maximum likelihood received sequence, as highlighted in [11] and [12].

\section{LINK CONTROL}

The adaptive link control algorithm specified in [R.05.08.] allows for the system to favour that specific traffic cell, which provides the highest probability of reliable communications associated with the lowest possible path loss. It also decreases interference with other cochannel users and, through dense RF frequency reuse, improves spectral efficiency, whilst maintaining an adequate communications quality and facilitates a reduction in power consumption, which is particularly important in hand-held MS's. The handover process maintains a call in progress as the MS moves between cells, or when there is an unacceptable degradation of quality caused by interference, in which case an intra-cell handover to another carrier in the same cell is performed. A radio link failure occurs when a call with an unacceptable voice or data quality cannot be improved either by RF power control or by handover. The reasons for the link failure may be loss of radio coverage or very high interference levels. The link control procedures rely on measurements of the received RF signal strength (RXLEV), the received signal quality (RXQUAL), and the absolute distance between base and mobile stations (DISTANCE).

The received signal level measurements are performed on the broadcast control channel (BCCH) carier which is continuously transmitted by the BS on all time slots and without variations of the RF level. A MS measures the received signal level from the serving cell and from the BS's in all adjacent cells by tuning and listening to their BCCH carriers. The rms level of the received signal is measured over a dynamic range of -103 to $-41 \mathrm{dBm}$ for intervals of one SACCH multiframe $(480 \mathrm{~ms})$. The received signal level is averaged over at least $32 \mathrm{SACCH}$ frames $(\approx 15 \mathrm{~s})$ and mapped to give RXLEV values between 0 and 63 to cover the range $-103 \ldots-41 \mathrm{dBm}$ in steps of $1 \mathrm{~dB}$. The RXLEV parameters are then coded into 6 bit words for transmission to the serving BS via the SACCH.

The received signal quality (RXQUAL) is estimated by measuring the BER before channel decoding, using the Viterbi channel equaliser's metrics and/or those of the Viterbi convolutional decoder. Eight values of RXQUAL span the logarithmically subdivided BER range of $0.2 \% \ldots 12.8 \%$ before channel decoding.

The absolute distance between base and mobile stations is measured using the "timing advance" parameter. The timing advance is coded as a 6 bit number corresponding to a propagation delay from 0 to $63 \cdot 3.69 \mu \mathrm{s}=$ $232.6 \mu \mathrm{s}$, characteristic of a cell radius of $35 \mathrm{~km}$.

While roaming, the MS needs to identify which potential target $\mathrm{BS}$ it is measuring and the $\mathrm{BCCH}$ carrier frequency may not be sufficient for this purpose, since in small cluster sizes the same $\mathrm{BCCH}$ frequency may be used in more than one surrounding cell. To avoid ambiguity a 6 bit Base Station Identity Code (BSIC) is transmitted on each $\mathrm{BCCH}$ carrier in the synchronisation burst(SB). Two other parameters transmitted in the $\mathrm{BCCH}$ data provide additional information about the BS. The binary flag called PLMN_PERMITTED indicates whether the measured $\mathrm{BCCH}$ carrier belongs to a PLMN which the MS is permitted to access. The second Boolean flag, CELL_BAR_ACCESS, indicates whether the cell is barred for access by the MS, although it belongs to a permitted PLMN. A MS in idle mode, i.e., after it has just been switched-on, or after it has lost contact with the network, searches all $125 \mathrm{RF}$ channels and takes readings of RXLEV on each of them. Then it tunes to the carrier with the highest RXLEV and searches for frequency correction bursts $(F C B)$ in order to determine whether or not the carrier is a BCCH carrier. If it is not, then the MS tunes to the next highest carrier, and so on, until it finds a $\mathrm{BCCH}$ carrier, synchronises to it and decodes the parameters BSIC, PLMN_PERMITTED and CELL_BAR_ACCESS in order to decide whether to continue the search. The MS may store the $\mathrm{BCCH}$ carrier frequencies used in the network accessed, in which case the search time would be reduced. The process described is summarised in the flowchart of Fig. 5.

The adaptive power control is based on RXLEV measurements. In every SACCH multiframe the BS compares the RXLEV readings reported by the MS, or obtained by the base station, with a set of thresholds. The exact strategy for RF power control is determined by the network operator with the aim of providing an adequate quality of service for speech and data transmissions and keeping interferences low. Clearly, 'adequate' quality must be achieved at the lowest possible transmitted power to keep cochannel interferences low, which implies contradictory requirements in terms of transmitted power. The criteria for determining the radio link failure are based on the measurements of RXLEV and RXQUAL performed by both the mobile and base stations and the procedures for handling link failures result in the re-establishment or the release of the call, depending on the network operator's strategy.

The handover process involves the most complex set of procedures in the radio link control. Handover decisions are based on results of measurements performed both by the base and mobile stations. The base station 


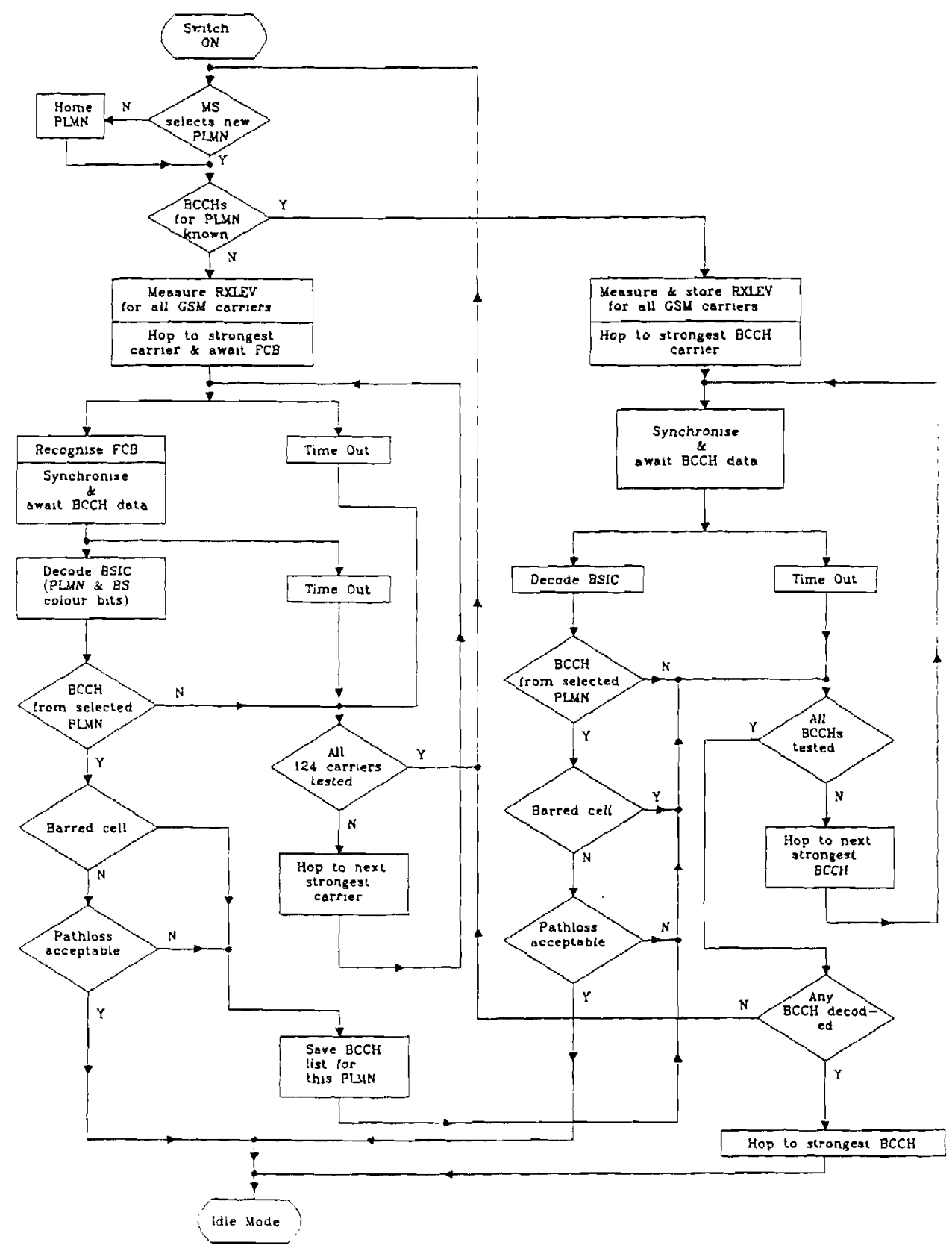

Fig. 5 - Initial call selection by the MS.

measures RXLEV, RXQUAL, DISTANCE, and also the interference level in unallocated time slots, while the MS measures and reports to the BS the values of RXLEV and RXQUAL for the serving cell, and RXLEV for the adjacent cells. When the MS moves away from the BS, the RXLEV and RXQUAL parameters for the serving station become lower, while RXLEV for one of the adjacent cells increases.

\section{DISCONTINUOUS TRANSMISSION}

Discontinuous transmission issues are standardised in
Recommendation [R.06.31], while the associated problems of voice activity detection are specified by [R.06.32.]. Assuming an average speech activity of $50 \%$ and a high number of interferers combined with frequency hopping to randomise the interference load, significant spectral efficiency gains can be achieved, when deploying discontinuous transmissions via decreasing interferences, while reducing power dissipation as well. Due to the reduction in power consumption full DTX operation is mandatory for MSs, but in BSs only receiver DTX functions are compulsory. Earlier adaptive VAD designs were proposed for PCM speech codecs. stationary hand sets and indoors background noise [ I5, 
16]. The fundamental problem is how to differentiate between speech and noise, while keeping false noise triggering and speech spurt clipping as low as possible. In vehicle-mounted MSs the severity of the speech/noise recognition problem is aggravated by the excessive vehicle background noise. This problem is resolved by deploying a combination of threshold comparisons and spectral domain techniques $[17,18]$, Another important associated problem is the introduction of noiseless inactive segments, which is mitigated by comfort noise insertion in these segments at the receiver, which is also addressed in $[17,18]$.

The DTX transmitter's operation relies on the VAD differentiating between speech and noise. If speech is deemed to be present, the VAD flag is set to one, while for noise $V A D=0$. If the VAD stops detecting speech, the DTX-handler does not immediately disable the speech transmission, but first enters the so-called Hangover (HGO) state, designed to prevent negligibly short silence periods from disabling transmissions as well as to minimise final talk spurt clipping. The HGO delay is of four speech frame durations, i.e., $4 \cdot 20=80 \mathrm{~ms}$ long, Even if the HGO delay has elapsed, the current frame must not be disabled, if it was stolen for the FACCH. With the HGO elapsed the system enters the Comfort Noise Update (CNU) state, where a so-called Silence IDentifier (SID) frame is sent to the receiver to update its comfort noise parameters. The SID frame is than sent during further silent periods in each SACCH multiframe, i.e., at intervals of $480 \mathrm{~ms}$. From the CNU state, if $\mathrm{VAD}=\mathrm{I}$ is encountered, the DTX transmitter state machine returns to its normal speech transmit (SPTX) state, otherwise it enters the Comfort Noise Computation $(\mathrm{CNC})$ state, sets the VAD flag to zero and disables transmissions. Detecting $\mathrm{VAD}=\mathrm{I}$ forces the system to SPTX state, while on VAD $=0$ further SID frames have to be transmitted in $\mathrm{CNU}$ mode, whenever a SACCH message is due for transmission.

The DTX receiver closely collaborates with the entire receiver, since it uses soft and hard decision information from the Viterbi channel equaliser, Viterbi channel decoder and cyclic error detecting block decoder to generate the so-called Bad Frame Indicator (BFI) flag. When the BFI flag signals a corrupted speech or SID frame, the Speech/noise Extrapolation (SE) functions are invoked to improve the perceived link quality. If, however, several adjacent frames are damaged, the received signal is gradually muted to zero. The interplay of system elements is completed by the comfort noise generator activated upon reception of SID frames for natural sounding Comfort Noise Insertion (CNI) in inactive speech intervals.

Firstly the DTX receiver decides, whether the received frame is a speech or an SID frame and, after evaluating the received signal quality, forms the pair (BFI, SID). The SID-detector is extremely reliable, since in SID frames all of the 95 FEC coded RPE excitation bits are set to zero at the transmitter and the re- ceived frame is only deemed to be an SID sequence, $\mathrm{i}$ at most 16 out of the 95 corresponding bits are non-zero The normal operation is described by (BFI $=0, \mathrm{SID}=$ 0 ), when an uncorrupted speech frame is received anc duly decoded by the speech decoder.

If a corrupted frame with $\mathrm{BFI}=1$ flag has arrived, irrespective of whether speech or noise is deemed to br present, the receiver switches into speech/noise extrapola tion mode to improve the subjective link assessment, ir case a single speech frame is corrupted or stolen by th FACCH. Several consecutive BFI $=1$ flags render the re ceiver to mute its output gradually to zero. When the link quality improves, $\mathrm{BFI}=0$ is encountered and upon SID = 0 the receiver returns to its normal speech decoding state.

Upon reception of an uncorrupted SID frame, th DTX receiver inserts comfort noise to the speech de coder, based on the noise spectral parameters (LARs received in the SID frame, which are updated by receiv ing fresh SID frames via the SACCH every $480 \mathrm{~ms}$ in each new multiframe. When detecting $B F I=0, S I D=0$ normal speech decoding is invoked, while if this frame happens to be corrupted, i.e., BFI $=1, \mathrm{SID}=0$, again the receiver enters the extrapolation mode (SE).

Experiments carried out by GSM have shown that silent gaps inserted by the DTX system are extremely an noying and degrade speech intelligibility. Best subjective and objective results are achieved, if comfort noise of ap propriately matched level and spectral envelope is insert ed and updated via sending an SID frame at each $480 \mathrm{~m}$ interval through the SACCH, when no speech is trans mitted. The 456 bit SID frame is a 'speech-like' fram transmitted every 24 - th $20 \mathrm{~ms}$ speech frame to charac terise the current background noise spectral envelope us ing the Logarithmic Area Ratio (LAR) parameters.

The level of the noise is represented by the subseg ment maxima computed by the speech encoder, but the Regular Excitation Pulses (RPE) are set to zero at the transmitter to aid the SID frame recognition at the re ceiver. The Long Term Predictor (LTP) is disabled by setting its gain to zero, while erratic noise level change are mitigated by limiting subsequent subsegment maxi ma increments to $50 \%$ of the previous maximum value Furthermore, the LARs and block maxima are averagec over the last four speech frames, before inclusion in ar SID frame. At the receiver the decoded LARs and block maxima are used with locally injected uniformly distrib uted pseudo-random RPE samples and grid positions to represent the background noise at the transmitter.

Whenever the BFI flag signals a corrupted speech o noise frame, the previous $20 \mathrm{~ms}$ frame is input to th speech decoder. This repetition is hardly perceptible, $\mathrm{i}$ only one frame is lost in every ten, but becomes inade quate when encountered more frequently. In subsequen corrupted frames therefore their level is gradually mut ed to zero by decreasing the maximum 64 -valued $(6 \mathrm{bi}$ logarithmically quantised) subsegment maxima eacl time by four. Hence it is set to zero in at most 16 subse quent $20 \mathrm{~ms}$ speech frames, i.e. in $320 \mathrm{~ms}$. 


\section{VOICE ACTIVITY DETECTION}

As we have seen in the previous section, the VAD imposed by Recommendation [R.06.32.] is the key element in controlling DTX functions. The VAD design deployed depends on the level and statistics of the typical background noise as well as on the implementation of the speech codec utilised, but differences between noise and speech properties can be exploited both in time [15] and frequency domain [17]. When using the GSM RPE codec in the high car-noise environment, a combination of spectral envelope, pitch periodicity and energy differences is utilised in the VAD decision process. The VAD's operation relies on an optimum compromise between minimum initial and final talk-spurt clipping and minimum on-air time, which are inherently contradictory requirements.

As seen in Fig. 6, the input SNR is first improved by 'adaptive noise filtering' using filter coefficients derived during noise-only periods, which is ensured by checking the 'stationary' and 'pitch' flags before filter coefficient adaptation. The filtered signal's energy $P_{\mathrm{VAD}}$ is then compared in the block 'VAD decision' against an adaptive threshold $T h_{\mathrm{VAD}}$ for a speech/noise decision represented by the $V_{V A D}$ flag. Finally, mid-spurt and end-spurt clipping of speech bursts is prevented by deploying a 'VAD hangover' (HGO) mechanism, delaying premature disabling of transmissions in case of mini-gaps during active speech spurts by $80 \mathrm{~ms}$, yielding the VAD flag.
The adaptive noise-filtering of the input signal is carried out using the set of filter coefficients $a_{i}, i=0 \ldots 8$ derived during noise-only periods. The filtered signal's energy $P_{Y A D}$ is compared against an adaptively adjusted threshold $T h_{\text {VAD }}$ to derive a speech/noise indicator signal VAD, which after being subjected to hangover yields the VAD flag utilised by the transmitter's DTX handler to enable/disable transmissions. The rest of the block diagram is concerned with the adaptive adjustment of the filter coefficients $a_{i}$ and that of the threshold $T h_{\text {VAD }}$.

The 'adaptive block filtering' operation of the 160 input signal samples $s(n)$ using an 8 -th order filter $a_{i}$ yields 168 samples of $s_{f}(n)$, as follows:

$$
\begin{gathered}
s_{f}(n)=\sum_{i=0}^{8} a_{i} s(n-i), \quad n=0 \ldots 167 \\
0 \leq(n-i) \leq 159
\end{gathered}
$$

The energy of the current $20 \mathrm{~ms}$ (160 samples) filtered input signal extended by the filter's 8 -sample memory is given by:

$$
P_{\mathrm{VAD}}=\sum_{n=0}^{167}\left(\sum_{i=0}^{8} a_{i} s(n-i)\right)^{2}, 0 \leq(n-i) \leq 159
$$

After expanding the operations in the expression of

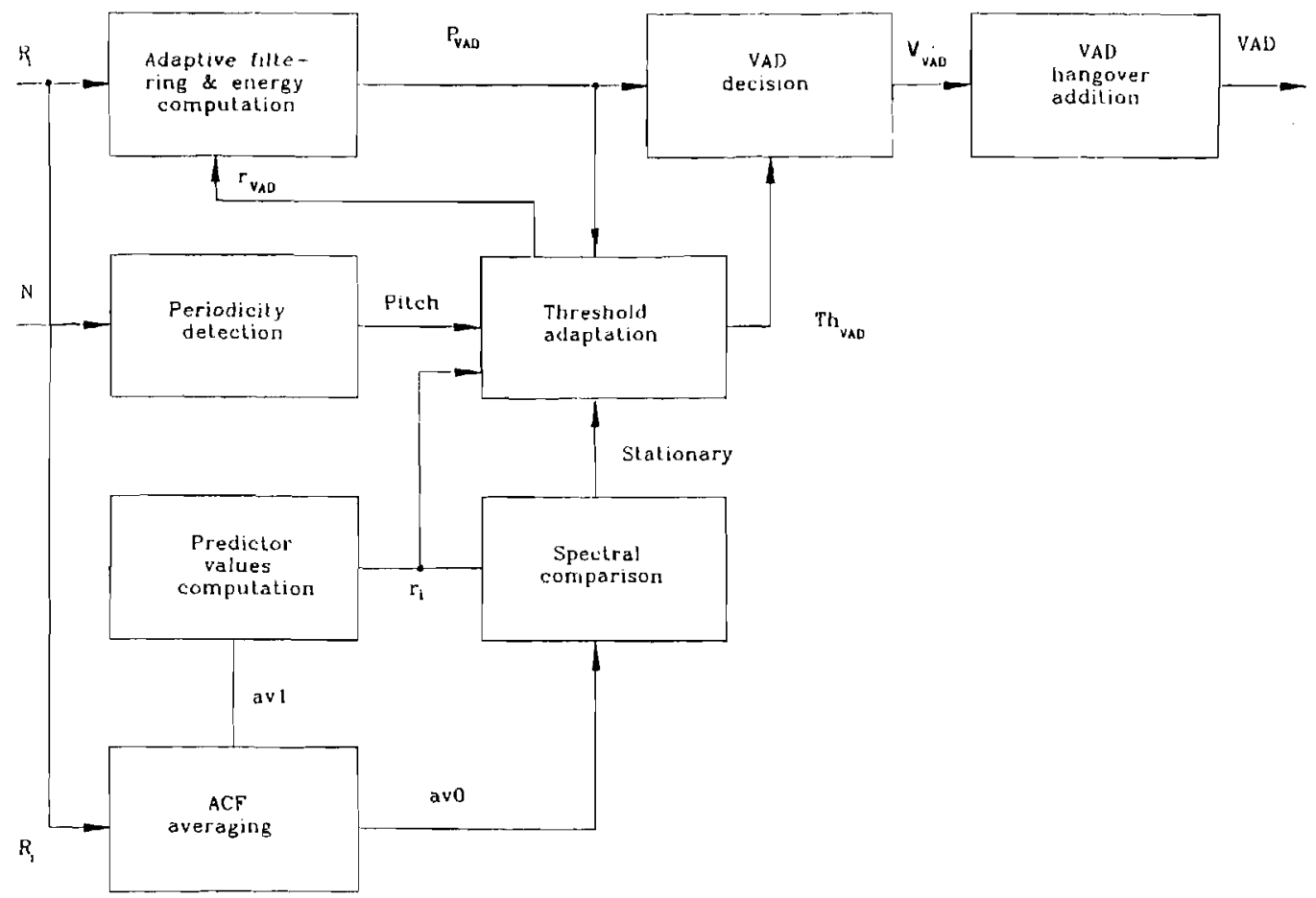

Fig. 6 - Functional block diagram of the VAD 
PVAD above and substituting the expressions

$$
R_{i}=\sum_{n=0}^{159} s(n) s(n-i), \quad i=0 \ldots 8, \quad 0 \leq(n-i) \leq 159
$$

and

$$
r_{i}=\sum_{k=0}^{8-i} a_{k} a_{k+i}, \quad i=0 \ldots 8
$$

for the input signal's and the filter coefficient's autocorrelations, respectively, we have:

$$
P_{\mathrm{VAD}}=r_{0} \cdot R_{0}+2 \sum_{i=1}^{8} r_{i} \cdot R_{i}
$$

The result of the 'VAD decision' is the Boolean flag $V_{\mathrm{VAD}}$, which is one if $P_{\mathrm{VAD}}>T h_{\mathrm{VAD}}$, zero otherwise. The HGO is implemented to prevent the VAD from prematurely curtailing the end of low-energy speech-spurts or removing short mid-speech silent gaps. The principle is that speech is continued to be transmitted for four more $20 \mathrm{~ms}$ frames, even if $V_{\mathrm{VAD}}=0$ indicates the presence of noise, in case at least three previous $20 \mathrm{~ms}$ speech segments were deemed to be present. Should, however $V_{\mathrm{VAD}}=\mathrm{I}$ be set during the $\mathrm{HGO}$ period, the 80 ms hangover period is resumed.

With the principles of VAD known, we now embark upon the description of the adaptive adjustment of the VAD-threshold, that of the filter coefficients $a_{i}$ and their correlations $r_{i}$. To get a stationary estimate of the input signal's statistics, each input signal autocorrelation coefficient $R_{i} i=0 \ldots 8$ is averaged over four frames, i.e., $80 \mathrm{~ms}$ to derive the averages $a v 0_{i}(n)=$ $\sum_{j=0}^{3} R_{i}(n-j), i=0 \ldots 8$, av $1_{i}(n)=a v 0_{i}(n-4), i=$ $0 \ldots 8$, where $n$ is the $20 \mathrm{~ms}$ frame index. The averaged autocorrelation coefficients $a v l_{i}(n)$ are input to the 'Predictor values computation' block using the Schur recursion [19] and the reflection coefficients $k_{i}(n)$ are computed exactly, as in the RPE-LTP speech codec. In determining the noise envelope's reflection coefficients av $1_{i}(n)$, the averages $i=0 \ldots 8$ are used, since $a v 0_{i}(n)$ might still contain the end of a speech burst. In a subsequent step the reflection coefficients $k_{i}$ are converted to simple finite impulse response (FIR) LPC filter coefficients $a_{i}, i=0 \ldots 8$ and their autocorrelation is computed as follows:

$$
r_{i}=\sum_{k=0}^{8-i} a_{k} \cdot a_{k+i}, \quad i=1 \ldots 8
$$

The LPC filter coefficient autocorrelations $r_{i}$, as well as the averaged input signal autocorrelation coefficients $a v 0_{i}$ are then compared using the simple distance measure $d_{m}$ defined as:

$d_{m}=r_{0} \cdot a v 0_{0}+2 \sum_{i=1}^{8} r_{i} \cdot a v 0_{i} / a v 0_{0}$

in order to derive a statistical similarity flag called 'Stationary' in the 'Spectral Comparison' block of Fig. 6. The spectral distance of the consecutive $20 \mathrm{~ms}$ input segments is evaluated by computing $d=\left(d_{m}\right.$ $\left.d_{m-1}\right)$, and Stationary $=1$ is set if $d<0.05$, i.e., the spectrum is deemed stationary, while Stationary $=0$, if the spectral difference $d \geq 0.05$, i.e., the spectrum is non-stationary.

The 'Threshold Adaptation' process is based on the input parameters Stationary, $r_{i}$ and $P_{\mathrm{VAD}}$ derived so far, as well as on the Boolean flag 'Pitch', indicating the presence of voiced input. This process has two outputvariables, the VAD decision threshold $T h_{\mathrm{VAD}}$ and the updated adaptive filter coefficient set $a_{i}, i=0 \ldots 8$ determining the updated set $r_{\mathrm{VAD}}=r_{i}$. The threshold adaptation updates $T h_{\mathrm{VAD}}$ every $20 \mathrm{~ms}$ in two basic scenarios. Whenever the signal energy is very low, a fixed value of $T h_{\mathrm{VAD}}=$ Constant is selected, since any further threshold adjustment would be unreliable due to the course quantisation at such low signal level. If, however, the signal energy is higher, further threshold adjustments in harmony with [R.06.32.] are possible. Finally, by forcing $r_{\mathrm{VAD}, i}=r_{i}, i=0 \ldots 8$, the set of filter coefficient correlations is updated for consecutive power computations.

The Pitch-flag, updated also every $20 \mathrm{~ms}$, becomes true if periodic input signal is detected, which is characteristic of speech but not noise. Note that the threshold $T h_{\mathrm{VAD}}$ is only updated, if the input is stationary but not periodic, i.e. when noise is deemed to be present.

In summary, the GSM VAD strikes a good compromise between lowest possible on-air time, i.e., activity and unobjectionable talk-spurt clipping. The typical channel activities vary from $55 \%$ in quiet locations through $60 \%$ in office noise to $65-70 \%$ in strong airport or railway station noise.

\section{CIPHERING}

The GSM communications security aspects are described in Recommendations $02.09,02.17,03.20$ and 03.21 , while an overview is given in [20]. The GSM security issues focus around the Authentication Centre (AUC) and the Subscriber Identity Module (SIM) received at subscription. The SIM is preferably a removable plug-in module with a Personal Identification Number (PIN). These features facilitate the production of identical hand sets with PIN protection against unauthorised use, while allowing GSM access through any GSM handset. The SIM contains, amongst a number of parameters, the International Mobile Subscriber Identity 
(IMSI), the Individual Subscriber Authentication Key $\left(K_{i}\right)$ and the Authentication Algorithm (A3). On attempting to access the PLMN the MS identifies itself to the network, receives a random number $(R)$, which together with $K_{i}$ is used to calculate the Signed response $(S)$ by invoking the confidential algorithm (A3): $S=\left[K_{i}\right.$ (A3) $R$ ]. The result $S$ is sent back to the network and compared with the locally computed version to authorise access. In addition to the random number $(R)$ the network sends a key number $\left(K_{n}\right)$ to the MS, which is related to the ciphering key $K_{c}$ and serves to avoid using different $K_{c}$ keys at the receiver and transmitter. This key number $K_{n}$ is then stored by the MS and is included in its first message to the network. Besides $S$, the MS computes the ciphering key $\left(K_{c}\right)$ using another confidential algorithm (A8) stored in the SIM, and the input parameters $K_{i}$ and $R: K_{c}=\left[K_{i}(\mathrm{~A} 8) R\right]$. The ciphering key $K_{e}$ is also computed in the network and hence no confidential information is sent unprotected via the radio path.

Once authentication is confirmed and both the network and the MS know $K_{c}$, the network issues a ciphering mode command and from now on all messages are ciphered at the transmitter and deciphered at the receiver, using the confidential algorithm (A5). Confidentiality is further enhanced by protecting the user's identity, when identification takes place, assigning a Temporary Mobile Subscriber Identity (TMSI) valid for a specific location area. This TMSI uniquely describes the IMSI in a specific location area, but outside the area it must be associated with the Location Area Identity (LAl). The network, more precisely the Visitor Location Register (VLR) keeps track of the TMSI-IMSI association and allocates a new TMSI in each new location area update procedure, i.e., in each new VLR.

The following representative example is provided to

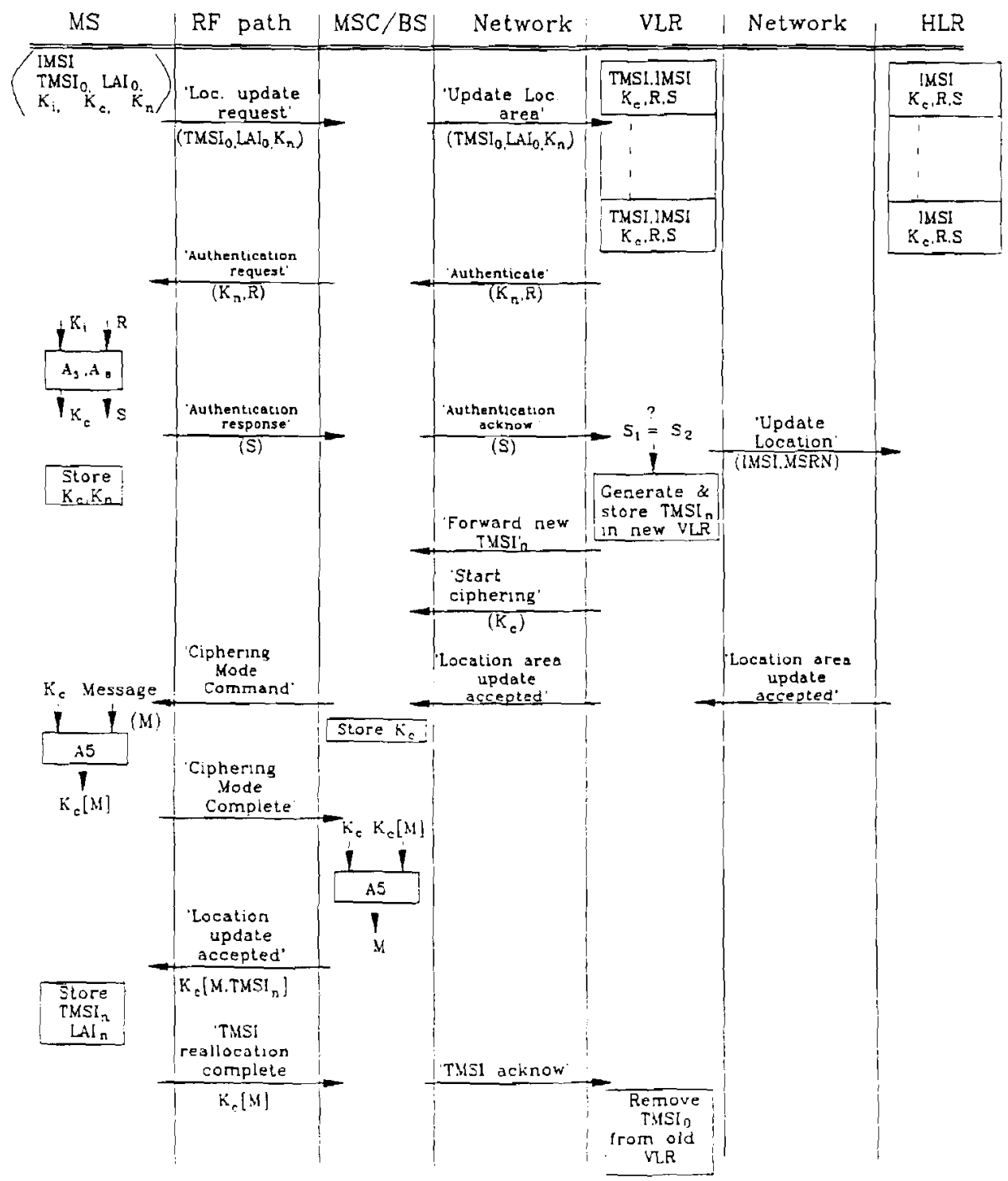

Fig. 7 - Location area update using the confidential algorithms A3, A.5 and A8. 
describe one out of a variety of specific scenarios, where the authentication and ciphering algorithms described are utilised. We assume that the MS is registered in the VLR and the TMSI is available. All required MS characteristics are stored in the VLR and identification is based on the LAI and TMSI parameters. As mentioned, authentication is carried out upon each location updating and the set [IMSI, TMSI $I_{n}, K_{c}$, $\left.K_{n}, R, S\right]$ is available in the VLR. The process is described with reference to Fig. 7 .

The MS stores its own set of [IMSI, TMSI, LAI, $K_{i}$, $K_{c}, K_{n}$ ] parameters and requests location update via the radio path by sending its [TMSI, $\mathrm{LAI}_{0}, K_{n}$ ] parameters to the MSC/BS. The MSC/BS forwards these via the network to the VLR, which issues an authentication request through the network to the MSC/BS and via the radio link to the MS by sending $K_{n}$ and $R$. The MS computes $K_{c}$ and $S$ from $K_{i}$ and $R$ utilising the algorithms $\mathrm{A} 3$ and $\mathrm{A} 8$, which are stored in its SIM. The signed response $S$ is transmitted back to the MSC/BS and from there via the network to the VLR, where authentication ensues by comparing the received and locally generated $S$ parameters. The VLR updates the MS's location in its own HLR at the appropriate IMSI entry and assigns a MS Roaming Number (MSRN). The MSRN stored in the MS's HLR is then used by incoming calls to find the MS and route the calls to the appropriate VLR, where the momentary TMSI and LAI parameters locate and identify the called subscriber.

Simultaneously, the VLR also generates the new TMSI $_{n}$ and forwards it to the MSC/BS and from now on TMSI $_{n}, \mathrm{LAI}_{n}$ and $K_{n}$ are used to identify the MS. The HLR acknowledges the location update to the VLR and to the MSC/BS that, in turn, issues a "ciphering mode command' to the MS. The MS responds with a 'ciphering mode complete' message and therefore ciphers all its messages by the algorithm A5 using $K_{c}$, while the MSC/BS deciphers and vice-versa. The MSC/BS informs the MS using a ciphered message that "location update is accepted' by the system and also sends out the new TMSI $_{n}$, which is acknowledged by the MS via sending the 'TMSI reallocation complete' message. Finally, this is accepted by the MSC/BS in that it sends a 'channel release' command to the MS and a 'TMSI acknowledge' to the old VLR to discard TMSI 0 .

\section{GSM SYSTEM PERFORMANCE}

The error rate performance of the RF subsystem is specified in Recommendation [R.05.05] for various propagation conditions, referred to as NAMEx, where $N A M E$ is the name of the propagation model and $x$ is the vehicle speed in $\mathrm{km} / \mathrm{h}$. The models for rural area, hilly terrain, typical urban area and the profile for equalisation testing are referred to as RAx, HTX, TUx and $E Q x$ channels, respectively. An additive white Gaussian noise channel, i.e., a static channel, is also considered. Depending on the type of traffic or control channel, the performance is described in terms of frame erasure rate (FER), bit error rate (BER), or residual bit error rate (RBER). The RBER is defined as the ratio of the number of errors detected due to unprotected Class 2 bits over the frames defined as "good", to the number of transmitted bits in the "good" frames, where a frame is deemed to be good for example, if the $(53,50)$ cyclic error detecting block code protecting the Class la speech bits does not indicate code overload.

In GSM parlance there are three different types of error rates. One is concerned with the conditions of operation at reasonable signal levels and in the absence of interference. Another applies when the receiver is operating with signal levels close to its noise floor, and the third category applies for operation in the presence of interference. The nominal error rates (NER) apply to propagation conditions, when there is no interference and the received RF signal level is equal to $-85 \mathrm{dBm}$. Under these conditions the chip error rate (channel $B E R$ ), which is equivalent to the bit error rate of the non-protected C2 bits of a full rate traffic channel for speech (TCH/FS), is specified as $\leq 10^{-4}$ for the static channel, $\leq 4 \times 10^{-3}$ for the rural channel RA250, $\leq 4 \times$ $10^{-4}$ for the typical urban channel TU 3 and $\leq 1 \%$ for the channel used for the equaliser testing.

Table 1-Reference sensitivity performance

\begin{tabular}{|lc|c|c|c|c|}
\hline \multirow{2}{*}{ Type of Channcl } & \multicolumn{4}{|c|}{ Propagation Conditions } \\
\cline { 3 - 6 } & (FER) & $0.1 \%$ & $4 \%$ & $4 \%$ & $6 \%$ \\
\hline SDCCH & Static & TU 50 & RA 250 & HT 100 \\
\hline RACH & (FER) & $0.1 \%$ & $10 \%$ & $10 \%$ & $10 \%$ \\
\hline SCH & (FER) & $1 \%$ & $15 \%$ & $15 \%$ & $15 \%$ \\
\hline TCH/F9.6, H4 & (BER) & $10^{-5}$ & $0.3 \%$ & $0.1 \%$ & $0.8 \%$ \\
\hline TCH/F4.8 & (BER) & & $10^{-4}$ & $10^{-4}$ & $10^{-4}$ \\
\hline TCH/F2.4 & (BER) & & $10^{-5}$ & $10^{-5}$ & $10^{-5}$ \\
\hline TCH/H2.4 & (BER) & & $10^{-4}$ & $10^{-4}$ & $10^{-4}$ \\
\hline TCH/FS & (FER) & $10^{-3}$ & $3 \%$ & $2 \%$ & $7 \%$ \\
\hline $\mathrm{Clb}$ & (RBER) & $0.4 \%$ & $0.2 \%$ & $0.2 \%$ & $0.5 \%$ \\
\hline $\mathrm{C} 2$ & (RBER) & $2 \%$ & $8 \%$ & $7 \%$ & $8 \%$ \\
\hline
\end{tabular}

Table 2 - Reference interference performance

\begin{tabular}{|lr|c|c|c|c|}
\hline \multirow{2}{*}{ Type of Channel } & \multicolumn{4}{|c|}{ Fiupagation Conditions } \\
\cline { 3 - 7 } & & TU 3 (No FH) & TU 3 (FH) & TU 50 & RA 250 \\
\hline SDCCH & (FER) & $8 \%$ & $4 \%$ & $4 \%$ & $4 \%$ \\
\hline $\mathrm{RACH}$ & (FER) & $12 \%$ & $12 \%$ & $12 \%$ & $10 \%$ \\
\hline $\mathrm{SCH}$ & (FER) & $15 \%$ & $15 \%$ & $15 \%$ & $15 \%$ \\
\hline $\mathrm{TCH} / \mathrm{F9} 9 / 6 / \mathrm{H} 4.8$ & (BER) & $1.5 \%$ & $0.3 \%$ & $0.3 \%$ & $0.2 \%$ \\
\hline $\mathrm{TCH} / \mathrm{F} 4.8$ & (BER) & & $10^{-4}$ & $10^{-4}$ & $10^{-4}$ \\
\hline $\mathrm{TCH} / \mathrm{F} 2.4$ & (BER) & & $10^{-5}$ & $10^{-5}$ & $10^{-5}$ \\
\hline $\mathrm{TCH} / \mathrm{H} 2.4$ & (BER) & & $10^{-4}$ & $10^{-4}$ & $10^{-4}$ \\
\hline $\mathrm{TCH} / \mathrm{FS}$ & (FER) & $7 \%$ & $2.5 \%$ & $3.5 \%$ & $3 \%$ \\
\hline $\mathrm{C} 1 \mathrm{~b}$ & (RBER) & $0.4 \%$ & $0.2 \%$ & $0.2 \%$ & $0.2 \%$ \\
\hline $\mathrm{C} 2$ & (RBER) & $8 \%$ & $8 \%$ & $8 \%$ & $8 \%$ \\
\hline
\end{tabular}


The reference sensitivity performance is the error rate performance when a received RF signal level is equal to the reference sensitivity level of $-102 \mathrm{dBm}$ for handportables and $-104 \mathrm{dBm}$ for all other mobile and base stations and when no interference is present. The reference sensitivity performance specifications are shown in Table 1 , while the reference interference figures are summarised in Table 2 for the different types of channels and propagation conditions.

The reference interference performance is the error rate limit when the wanted input RF signal level is -85 $\mathrm{dBm}$ and a random GSM modulated interfering signal is present. The signal-to-interference ratio is $\leq 9 \mathrm{~dB}$, while the signal-to-adjacent channel ratios at 200 and $400 \mathrm{kHz}$ from the carrier are $<-9 \mathrm{~dB}$ and $-41 \mathrm{~dB}$, respectively. This interference ratio is called the "reference interference ratio" and is identical for all types of base and mobile stations. In the tests, the wanted and interfering signals are subject to the same propagation profiles, and when frequency hopping is used, they have the same hopping sequence. Under these conditions the error rates for the various types of channels and propagation conditions satisfy the limits shown in Table 2.

The performance of a complete GSM speech channel simulator has been reported in [13] for the various GSM channel models using vehicular speeds ranging from 0 $\mathrm{km} / \mathrm{h}$ (AWGN) through pedestrians walking at $3 \mathrm{~km} / \mathrm{h}$ to $250 \mathrm{~km} / \mathrm{h}$ high-speed trains. The standardised typical urban (TU), rural area (RA), hilly terrain (HT) and equaliser test (EQ) GSM impulse responses were deployed, as characterised earlier in Fig. 2 of section 3 for

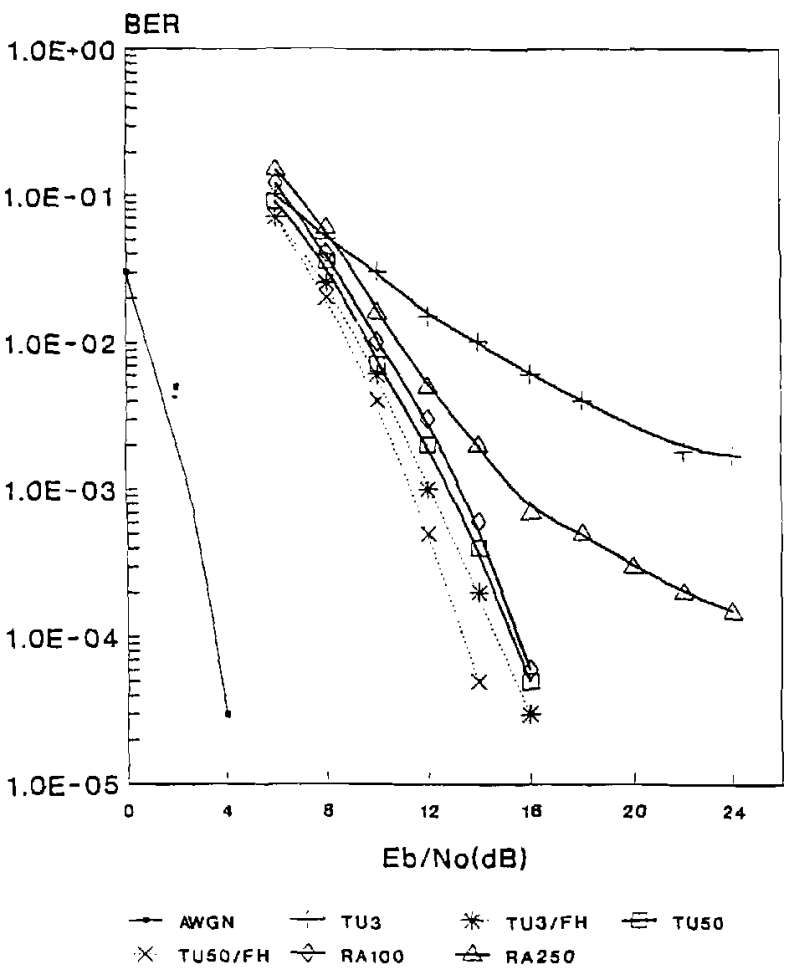

Fig. 8 - Speech Cl BER vs. $E_{1} / N_{0}$ performance, Hodges et. al. [13]. various mobile speeds described in terms of $\mathrm{km} / \mathrm{h}$ figures associated with the GSM channel acronyms. In some cases also the stationary AWGN results as flat Rayleigh-fading (RAY) results are quoted. For slowly walking pedestrians results are portrayed both with and without frequency hopping $(\mathrm{FH})$. The concatenated coded $\mathrm{Cl}$ speech BER vs. $E_{b} / N_{0}$ results are reproduced in Fig. 8, where we observe virtually error free operation for the AWGN channel for $E_{b} / N_{0}$ in excess of $4 \mathrm{~dB}$ and for most of the fading channels above $12 \mathrm{~dB}$. When using the typical urban (TU3) channel for pedestrians, the MSs are idling in deep fades and so the interleaving memory is not sufficiently long to randomise error bursts before channel decoding, which yields a high residual BER. This is seen being effectively combated by FH. The higher residual BER of the rural area (RA250) channel is due to the higher Doppler shift and lack of 'diversity effect' engendered by the sharply decaying impulse response. The unprotected $\mathrm{C} 2$ bits have a high residual BER in Fig. 9, which is unaffected by FH. In fact, this residual C2 BER is higher than that of the VE implementations proposed in $[9,10]$. Similar tendencies are recognised as regards to Frame Error Rates (FER) depicted in Fig. 10. The interference resistance of the system expressed in carrier to interference ratio [C/I $(\mathrm{dB})$ ] is also characterised in [13], which is again similar in its tendencies to the noise resistance, as seen in Fig. 11, 12 and 13.

All in all, the reported VE implementations reduce the channel BER to values sufficiently low for the concatenated channel coding/interleaving scheme to

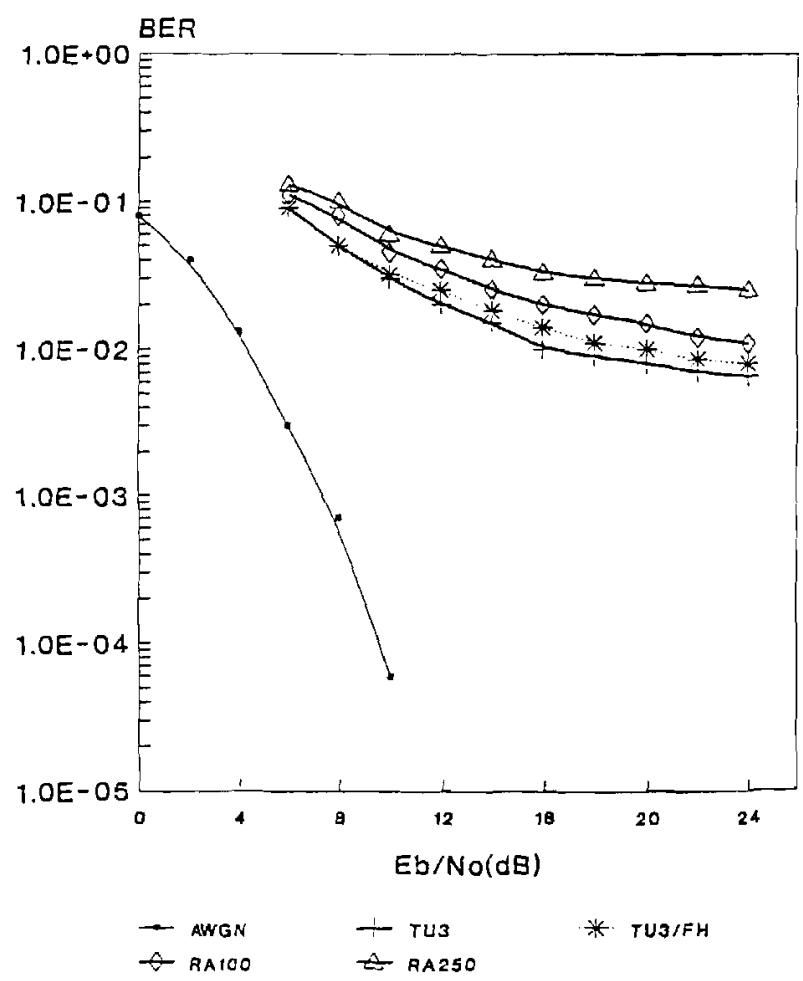

Fig. $\boldsymbol{Y}$ - Speech $C 2$ BER vs. $E_{h} / N_{0}$ performance, Hodges et. al. [13]. 

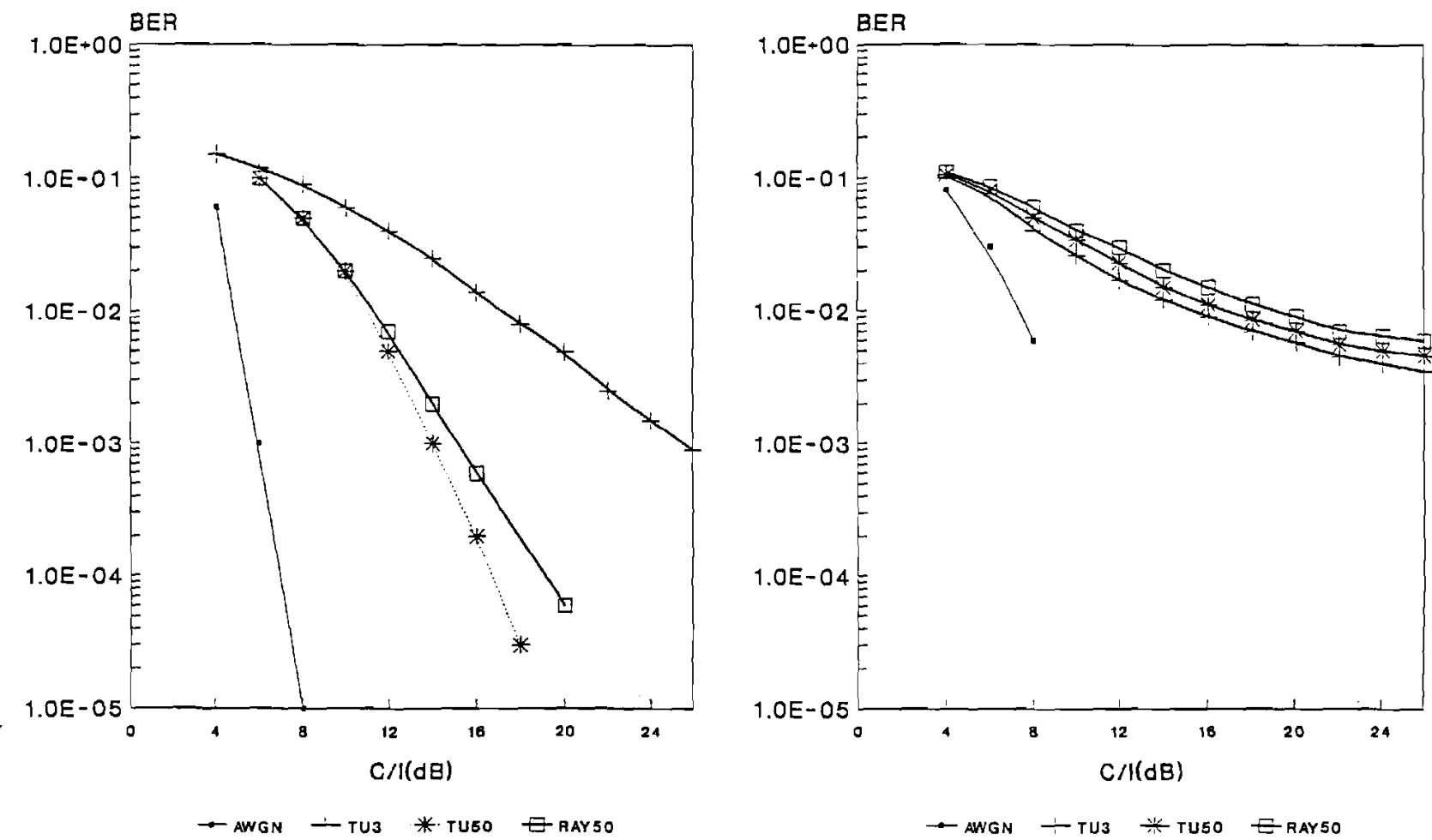

Fig. 10 - Speech FER vs. $E_{0} / N_{0}$ performance. Hodges et. al. [13].

Fig. 12 - Speech C2 BER vs. C/I performance, Hodges et. al. [13].
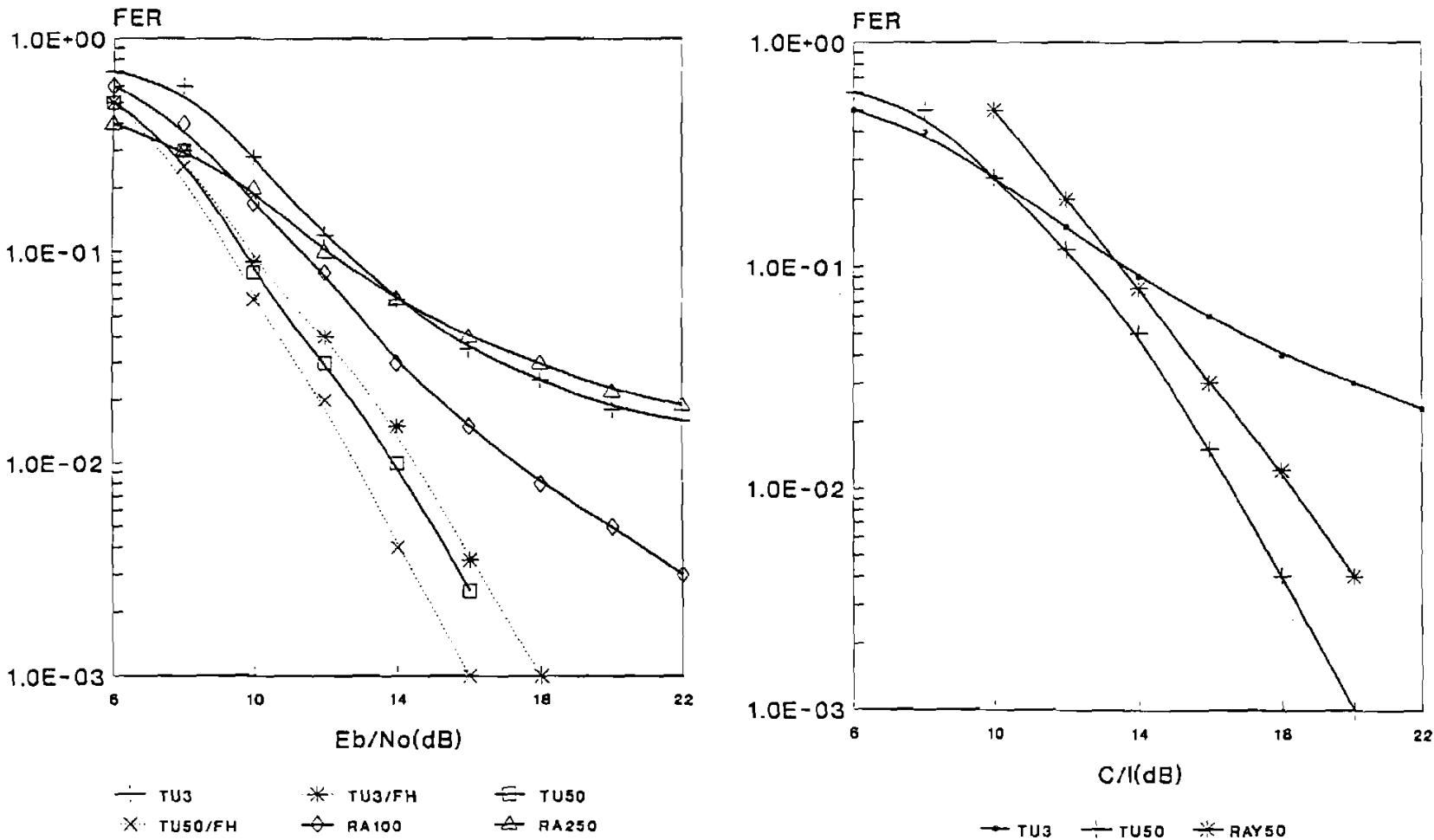

Fig. 11 - Speech CI BER vs. C/l performance, Hodges et. al. [13].

Fig. 13 - Speech FER vs. C/I pertormance, Hodges et. al. [13]. 
remove most of the errors for $E_{b} / N_{0}$ and $\mathrm{C} / \mathrm{I}$ ratios in excess of 12-14 dB, a value providing higher robustness and spectral efficiency than current analogue systems.

\section{CONCLUSIONS}

The Pan-European system was portrayed for the practioner in a deductive approach. The mapping of logical traffic and control channels onto physical channels was detailed through describing the source codecs, channel codecs and interleavers. Their transmission using GMSK via wideband channels, assisted by Viterbiequalization and soft-decision Viterbi channel decoding yields virtually unimpaired speech quality for channel SNRs and signal to interference ratios in excess of 12 $14 \mathrm{~dB}$. The $1.35 \mathrm{bit} / \mathrm{s} / \mathrm{Hz}$ spectral efficiency of the modem, the intelligent adaptive transmit power control and discontinuous transmissions all contribute to the high system bandwidth efficiency achieved. The key system features are summarised in Table 3.

Table 3 - Summary of GSM features

\begin{tabular}{|l|c|}
\hline System Feature & \\
\hline Uplink bandwidth & $890-915=25 \mathrm{MHz}$ \\
\hline Downlink bandwidth & $935-960=25 \mathrm{MHz}$ \\
\hline Total GSM bandwidth & $50 \mathrm{MHz}$ \\
\hline Carrier spacing & $200 \mathrm{kHz}$ \\
\hline No. of RF carriers & 125 \\
\hline Multiple access & TDMA \\
\hline No.of users/carriers & 8 \\
\hline Total No. of channels & 1000 \\
\hline TDMA burst rate & $271 \mathrm{kbit} / \mathrm{s}$ \\
\hline Modulation & $1.35 \mathrm{bit} / \mathrm{s} / \mathrm{Hz}$ \\
\hline Bandwidth efficiency & yes \\
\hline Channel equaliser & $13 \mathrm{kbit} / \mathrm{s}$ \\
\hline Speech coding rate & $22.8 \mathrm{kbit} / \mathrm{s}$ \\
\hline FEC coded speech rate & Gmbedded block $/ \mathrm{convolutional}$ \\
\hline FECcoding & $217 \mathrm{hops} / \mathrm{s}$ \\
\hline Frequency hopping & yes \\
\hline DTX and VAD & $35 \mathrm{~km}$ \\
\hline Maximum cella radius & \\
\hline
\end{tabular}

\section{Acknowledgement}

This paper is the crystallization of the GSM/ETSI standards as well as of numerous excellent papers refer- enced, whence the authors are indebted to all 'GSM contributors'.

Manuscript received on June 6, 1993

\section{REFERENCES}

[1] R. Steele, et.al: Digital mobile radio communtication. ISBN 07273 - 1406 - 8, Pentech Press, 1992.

[2] Proceedings of the Nordic Seminar on Digital Land Mobile Radiocommunication (DMR). February 1985, Espoo, Finland.

[3] Proceedings of the Second Nordic Seminar on Digital Land Mobile Radiocommunication (DMRII). October 1986, Stockholm, Sweden.

[4] Proceedings of the International Conference on Digital Land Mobile Radiocommunication (ICDMC). June/July 1987, Venice, Italy.

[5] Proceedings of Digital Cellular Radio Conference. Oct. 12-14. 1988, Hagen, FRG.

[6] Group Speciale Mobile. ETSI Recommendation.

[7] L. B. Lopes: GSM radio link simulation IEE Colloquium. University research in Mobile Radio, 1990, p. 5/1-5/4.

[8] J. Cheng, R. Steele; Modified Viterbi equaliser for mobile radio channels having large multi-path delay. "Electronics Letters", Vol. 25, No. 19, p. 1309-131I.

[9] N. S. Hoult, C. A. Dace, A. P. Cheer: Implementation of an equaliser for the GSM system. Proc. of the 5th Int. Conf. on Radio Receivers Associated Systems, 24-26 July, 1990, Cambridge, U.K.

[10] R. D'Avella, L. Moreno, M. Sant'Agostino: An adaptive MLSE receiver for TDMA digital mobile radio. "IEEE Joumal on Selected Areas in Communications", Vol. 7, No. 1, Jan. 1989, p. 122-129.

[11] J. C. S. Cheung: Receiver techniques for wideband time division multiple access mobile radio systems. PhD Mini-Thesis, 1990, Univ. of Southampton.

[12] J. B. Anderson, T. Aulin, C. E. Sundberg: Digital phase modulation. Plenum Press, 1986.

[13] M. R. L. Hodges, S. A. Jensen, P. R. Tattersall: Laboratory testing of digital cellular radio systems. "BTRL Journal", Vol. 8, No. 1, Jan. 1990, p. 57-66.

[14] D. J. Targett, H. R. Rast: Handover-enhanced capabilities of the GSM system. Proc of Digital Cellular Radio Conference, Oct. 12-14.1988, Hagen, FRG, p. 3C/1-3C/11.

[15] E. Bacs, L. Hanzo: A simple real-time adaptive speech detector for SCPC systems. Proc of ICC 85, Chicago, p. 1208-1212.

[16] J. A. Jankowski: A new digital voice-activated switch. "Comsat Tech. Journal", Vol. 6, No. 1, Spring 1976, p. 159-170

[17] D. K. Freeman, G. Cosier, C. B. Southcott, 1. Boyd: The voice activity detector for the Pan-European digital cellular mobile telephone service. Proc. of ICASSP 89, Glasgow, p. 369-372.

[18] S. Hansen: Voice Activiry Detection (VAD) and the operation of Discontinuous Trunsmission(DTX) in the GSM system. Proc. of Digital Cellular Radio Conference, Oct. 12-14, 1988, Hagen, FRG, p. 2b/1-2b/14.

[19] P. C. J. Arend: Security aspects and the implementation in the GSM system. IBID., p. $4 \mathrm{a} / 1-4 \mathrm{a} / 7$.

[20] J. Schur: Über Poten-reihen, die in Innern des Einheitskreises beschränkt sind. "Journal für die reine und angewandte Mathematik". Vol. Bd 147, p. 205-232, 1917. 